\title{
Assisted reproductive technology in South Africa: First results generated from the South African Register of Assisted Reproductive Techniques
}

\author{
S J Dyer, T F Kruger
}

Objective. We present the first report from the South African Register of Assisted Reproductive Techniques.

Methods. All assisted reproductive technology (ART) centres in South Africa were invited to join the register. Participant centres voluntarily submitted information from 2009 on the number of ART cycles, embryo transfers, clinical pregnancies, age of female partners or egg donors, and use of fertilisation techniques. Data were anonymised, pooled and analysed.

Results. The 12 participating units conducted a total of 4512 oocyte aspirations and 3872 embryo transfers in 2009, resulting in 1303 clinical pregnancies. The clinical pregnancy rate (CPR) per aspiration and per embryo transfer was $28.9 \%$ and $33.6 \%$, respectively. Fertilisation was achieved by intracytoplasmic sperm injection in two-thirds of cycles. In most cycles, $1-2$ embryos or blastocysts were transferred. Female age was inversely related to pregnancy rate.

Conclusion. The register achieved a high rate of participation. The reported number of ART cycles covers approximately $6 \%$ of the estimated ART demand in South Africa. The achieved CPRs compare favourably with those reported for other countries.

S Afr Med J 2012;102:167-170.
Assisted reproductive technology (ART) has become increasingly globalised, with approximately $80 \%$ of the world's population living in countries where ART is practised. ${ }^{1}$ The growing impact of ART on human reproduction has resulted in a call for data on its efficacy and safety. Information on treatment safety and outcome is relevant to patients, the general public, healthcare planners, funders, and ART units. ${ }^{2}$ Relevant information cannot, however, be extrapolated from research trials; rather, a data registry is required.

Efficacy and safety data are collected at national, regional and global level. The World Collaborative Report on ART gave an account of approximately 930000 initiated ART cycles from 54 countries, representing an estimated $60-70 \%$ of global ART activity. ${ }^{3}$ These data were derived from all world regions except sub-Saharan Africa, where no country had monitored ART. To close the information gap in South Africa, the South African Society of Reproductive Medicine and Gynaecological Endoscopy (SASREG) established a sub-committee in 2007 to institute voluntary, national, anonymous ART data monitoring. We report the results of the first data collection.

ART is defined as 'all treatments or procedures that include the in vitro handling of both human oocytes and sperm, or embryos, for the purpose of establishing a pregnancy.' ${ }^{4}$ This does not include artificial insemination. Oocytes are aspirated from ovaries (usually after ovarian stimulation), incubated in culture medium and fertilised by the addition of spermatozoa - a process termed in vitro fertilisation (IVF). If sperm quality is poor, sperm are injected into oocytes by intracytoplasmic sperm injection (ICSI). Successful fertilisation via

Department of Obstetrics and Gynaecology, Groote Schuur Hospital and Faculty of Health Sciences, University of Cape Town s J Dyer, MB ChB, FCOG (SA), MMed, PhD

Department of Obstetrics and Gynaecology, Faculty of Health Sciences, Stellenbosch University

T F Kruger, MB ChB, MPharMed (Clin Phar), MMed (O\&G), MD, FRCOG
IVF or ICSI gives rise to human zygotes which develop into embryos then blastocysts; either of these is introduced into the recipient through trans-cervical intra-uterine transfer. No law or guideline in South Africa currently stipulates how many embryos/blastocysts may be transferred.

Biochemical pregnancy is characterised by raised serum human chorionic gonadotrophin (hCG), detected as early as 10 days after initial aspiration. Pregnancy may progress to clinical pregnancy - either viable intrauterine pregnancy or early pregnancy loss (miscarriage and ectopic pregnancy). A percentage of clinical pregnancies will result in live births.

Published reports on national, regional and global ART data monitoring - including this manuscript - have not undergone ethics committee review or approval. ${ }^{3,5-7}$ The reported data are anonymous and were collected on a voluntary basis. The data were pooled and de-linked from the source databases; therefore, there is no risk to patient confidentiality.

\section{Methods}

All known ART units were invited to join the South African Register of Assisted Reproductive Techniques (SARA). Consultation on the type of data to collect and the data collection means was informed by international recommendations and consensus documents. ${ }^{2,4}$ International collaboration was developed with the International Committee Monitoring ART (ICMART) and the Latin American Registry of ART (Registro Latinoamericano de Reproducción Asistida) (RLA).

National data collection was initiated in a 2-step approach. In the first step, 2009 data were collected using a Microsoft Excel spreadsheet: data collected pertained to the number of cycles, embryo transfers, clinical pregnancies, age of female partner and egg donor, and use of fertilisation techniques (IVF and ICSI). Only fresh embryo transfers were included. Procedures and outcomes were defined in keeping with the ICMART-WHO glossary. 'Aspiration' was defined as attempted oocyte retrieval irrespective of the outcome. In cycles where both IVF and ICSI were used, the technique that gave rise to the embryo(s) transferred was captured; ICSI was recorded as the default if embryos from both techniques were transferred. Donor egg aspirations, embryo transfers and clinical pregnancies were 
all recorded according to the age of the egg donor, and not that of the recipient. The number of embryos or blastocysts transferred in each cycle was captured, but embryo and blastocyst transfers were not reported separately. Only clinical pregnancies were captured defined as 'any pregnancy with clinical products of conception or ultrasonographic evidence of pregnancy' (this excludes biochemical pregnancies). It was anticipated that these data could be provided by most of the SARA participating units. In the second step, a software programme was developed in collaboration with the RLA for future online reporting of more extended data.

In March 2010 a first call for data was sent to 16 South African ART physicians and units. Two units were excluded as they had previously declined participation. By the end of 2010, 12 units had sent their 2009 summary data to the SASREG secretariat. Data were sent electronically with password protection to 2 non-clinical members of the secretariat (data managers), whose role was to protect data confidentiality, test for mathematical errors, and pool data without error. No further attempt was made to validate data devoid of mathematical error. The pooled data were analysed by the authors.

\section{Results}

Data were received from 12 participant ART units. One unit was only able to provide data for July - December 2009 for operational reasons. Mathematical errors were found in the data from 2 units; these were rectified following consultation between the units and the data managers.

A total of 4512 aspirations and 3872 embryo transfers were conducted, resulting in 1303 clinical pregnancies; the clinical pregnancy rate (CPR) per aspiration and per embryo transfer was $28.9 \%$ and $33.6 \%$, respectively.

Nine units provided information on: (i) fertilisation technique (i.e. IVF and ICSI), (ii) age of female partner or egg donor, and (iii) number of embryos/blastocysts transferred. These 9 units undertook 3305 aspirations and 2813 embryo transfers, resulting in 974 clinical pregnancies. ICSI accounted for $62.3 \%$ of aspirations, $63.2 \%$ of embryo transfers and $59.1 \%$ of clinical pregnancies. Table 1 summarises the data on IVF-related procedures and outcomes according to age of female partner or egg donor. Similarly, Table 2/ Fig. 1 shows the number of embryos/blastocysts transferred after IVF.

Tables 3 and 4 summarise the data pertaining to ICSI-related procedures and outcomes, and the number of embryos/blastocysts transferred, respectively. Fig. 2 depicts the information contained in Table 4.

Table 1. In vitro fertilisation (IVF): number of procedures and outcomes according to age of female partner or egg donor

\begin{tabular}{lllll}
\hline & $<35$ & $35-39$ & $>39$ & \\
IVF & years & years & years & Total \\
\hline Aspirations $(N)$ & 686 & 367 & 191 & 1244 \\
Embryo transfers $(N)$ & 586 & 298 & 150 & 1034 \\
Clinical pregnancies $(N)$ & 256 & 104 & 38 & 398 \\
$\mathrm{CPR}^{\dagger} /$ aspiration $(\%)$ & 37.1 & 28.3 & 19.8 & 31.9 \\
$\mathrm{CPR} /$ embryo transfer $(\%)$ & 43.7 & 34.9 & 25.3 & 38.5 \\
$\begin{array}{l}\text { * Egg donor procedures and outcomes are captured according to age of female partner or } \\
\text { egg donor, and not recipient age. }\end{array}$ & & & &
\end{tabular}

Table 2. Number of embryos/blastocysts transferred after

IVF, according to age of female partner or egg donor

\begin{tabular}{lllll}
\hline & $\begin{array}{l}<5 \text { years } \\
N(\%)\end{array}$ & $\begin{array}{l}35-39 \\
\text { years } \\
N(\%)\end{array}$ & $\begin{array}{l}>39 \\
\text { years } \\
N(\%)\end{array}$ & $\begin{array}{l}\text { Total } \\
\text { transfers } \\
N(\%)\end{array}$ \\
\hline $\begin{array}{l}1 \text { embryo/ } \\
\text { blastocyst } \\
2 \text { embryos/ } \\
\text { blastocysts }\end{array}$ & $60(10.2)$ & $51(17.1)$ & $37(24.6)$ & $148(14.3)$ \\
$\begin{array}{l}3 \text { embryos/ } \\
\text { blastocysts }\end{array}$ & $97(16.5)$ & $80(26.8)$ & $46(30.6)$ & $223(21.6)$ \\
$\begin{array}{l}4 \text { or more } \\
\text { embryos/ }\end{array}$ & $30(5.1)$ & $32(10.7)$ & $23(15.3)$ & $85(8.2)$ \\
blastocysts & & & & \\
Total transfers & $586(100)$ & $298(100)$ & $150(100)$ & 1034
\end{tabular}

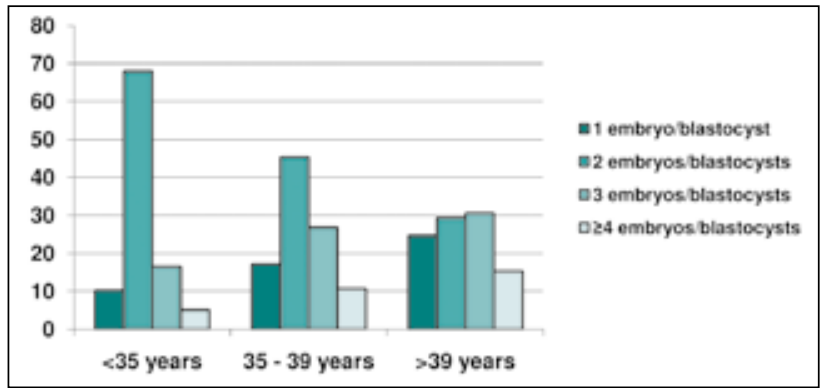

Fig. 1. Distribution of the different number of embryos/blastocysts transferred following IVF, according to age of female partner or egg donor. The 3 age categories are shown on the $x$-axis. The $y$-axis captures percentage of transfers with a given number of embryos/blastocysts per age group; $100 \%=$ all embryo transfers in the age group.

Table 3. Intracytoplasmic sperm injection (ICSI): number of procedures and outcome, according to age of female partner or egg donor

\begin{tabular}{lllll}
\hline & & $35-39$ & $>39$ \\
ICSI & $<35$ years & years & years & Total \\
\hline Aspirations $(N)$ & 992 & 702 & 367 & 2061 \\
Embryo transfers $(N)$ & 913 & 595 & 271 & 1779 \\
Clinical pregnancies $(N)$ & 328 & 189 & 59 & 576 \\
CPR per aspiration (\%) & 33 & 26.9 & 16 & 27.9 \\
CPR per embryo & 35.9 & 31.8 & 21.8 & 32.2 \\
transfer $(\%)$ & & & & \\
& & & &
\end{tabular}

\section{Discussion}

This is the first report from South Africa and sub-Saharan Africa on national anonymous monitoring of ART. Approximately 70\% of ART units participated, reflecting a willingness to collaborate to obtain national ART data. Participation is expected to increase as some nonparticipating units have announced their intention to join SARA, and others may become convinced of the benefit from national data monitoring.

Optimal population ART coverage has been estimated at 1500 cycles per million individuals per year. ${ }^{8,9}$ South Africa has a large unmet ART need: based on a South African population of 44.8 million people (Census 2001), ${ }^{10}$ the 4500 reported aspirations 


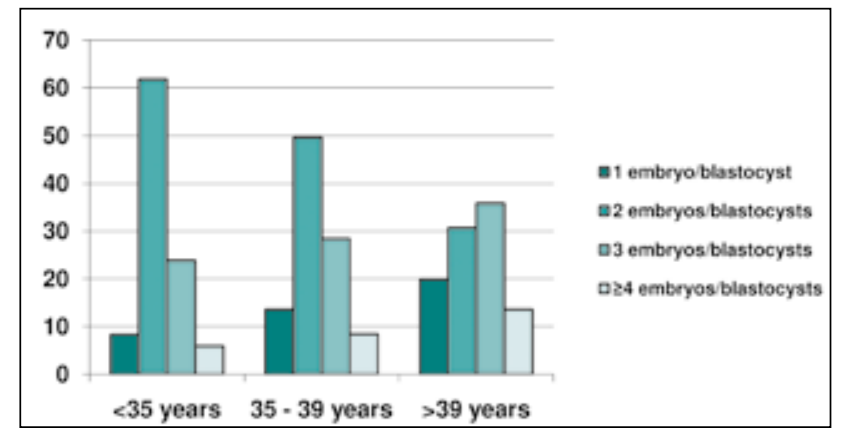

Fig. 2. Distribution of the different number of embryos/blastocysts transferred after ICSI, according to age of female partner or egg donor. The 3 age categories are shown on the $x$-axis. The $y$-axis captures percentage of transfers with a given number of embryos/blastocysts per age group; $100 \%=$ all embryo transfers in the age group.

\begin{tabular}{|c|c|c|c|c|}
\hline & $\begin{array}{l}<35 \text { years } \\
N(\%)\end{array}$ & $\begin{array}{l}35-39 \\
\text { years } \\
N(\%)\end{array}$ & $\begin{array}{l}>39 \text { years } \\
N(\%)\end{array}$ & $\begin{array}{l}\text { Total } \\
\text { transfers } \\
N(\%)\end{array}$ \\
\hline $\begin{array}{l}1 \text { embryo/ } \\
\text { blastocyst }\end{array}$ & $76(8.3)$ & $81(13.6)$ & $54(19.9)$ & 211 (11.9) \\
\hline $\begin{array}{l}2 \text { embryos/ } \\
\text { blastocysts }\end{array}$ & $565(61.8)$ & $295(49.6)$ & $83(30.6)$ & $943(53)$ \\
\hline $\begin{array}{l}3 \text { embryos/ } \\
\text { blastocysts }\end{array}$ & $218(23.8)$ & $169(28.4)$ & $97(35.8)$ & $484(27.2)$ \\
\hline $\begin{array}{l}4 \text { or more } \\
\text { embryos/ } \\
\text { blastocysts }\end{array}$ & $54(5.9)$ & $50(8.4)$ & 37 (13.6) & $141(7.9)$ \\
\hline $\begin{array}{l}\text { Total transfers } \\
N(100 \%)\end{array}$ & $913(100)$ & $595(10.7)$ & $271(100)$ & $\begin{array}{l}1779 \\
(100)\end{array}$ \\
\hline
\end{tabular}

represent approximately $6 \%$ of optimal ART coverage. Barriers to ART include the cost, which is poorly - if at all - covered by private health insurances; access in the public health sector is limited to very few institutions, and patients usually have to contribute to the costs. Out-of-pocket payment for a standard IVF cycle with standard ovarian stimulation ranges from approximately R10 000 (subsidised care in the public sector) to R35 000 (private sector care). The impact of appropriate funding for ART was illustrated in Germany, where a reduction of third-party ART reimbursement in 2004 resulted in almost a 50\% drop in annual treatment cycles. ${ }^{5}$ Geographical barriers also exist: South Africa's limited number of ART units are found in urban areas. Additional constraints to the extensive uptake of ART include a lack of knowledge, religious beliefs, and a lack of trust in a costly technique of varying success, which is not always outlined in a consistent or transparent manner.

The CPR per aspiration was $31.9 \%$ for IVF and $27.9 \%$ for ICSI. Evidently, the CPR per embryo transfer was higher $38.5 \%$ for IVF and $32.2 \%$ for ICSI), as some aspirations were unsuccessful, and in certain cycles none of the retrieved oocytes developed into embryos. These results are reassuring and reflect a sound efficacy of ART in South Africa. They are within the mid-range of internationally reported data, although any such comparison is fraught with difficulty because of inherent differences in the collected data. The World Report on ART monitoring, which captured data from 2003, documented a variable IVF CPR per aspiration of between 11.8\% (Guatemala) and 41.7\% (Taiwan), with most countries situated between $20 \%$ and $35 \%$. The global CPR per aspiration was $28.6 \%$ for IVF and $29.4 \%$ for ICSI. ${ }^{3}$ Similar results were reported from the European register: approximately 450000 ART cycles conducted in 2006 were recorded (including frozen embryo replacement), resulting in a CPR per aspiration of $29 \%$ (IVF) and $29.9 \%$ (ICSI). ${ }^{5}$ Large variations were noted between countries, including differences in national coverage of ART, age of the women treated, number of embryos/blastocysts transferred, and multiple pregnancy rates.

ICSI, rather than standard IVF, was performed in $62.3 \%$ of aspirations. Very similar rates have been reported from the USA, Europe and Australia in 2006. ${ }^{5}$ Moreover, regional and international registers have observed the growing use of ICSI, attributable to changes in professional practice, rather than an overall increase in male factor infertility. ${ }^{5,11}$ It will be interesting to monitor this trend in South Africa, with the knowledge that ICSI does not improve the outcome of ART for non-male infertility. ${ }^{11}$

Approximately $50 \%$ of women undergoing IVF and ICSI were under the age of 35 , representing almost two-thirds of all clinical pregnancies reported; 15 - 17\% were $\geq 40$ years, with approximately $1 / 10$ pregnancies occurring in this age-group. It is encouraging that most patients underwent ART at a young age, when the prognosis for pregnancy is best. A CPR per embryo transfer of $25.3 \%$ (IVF) and $21.8 \%$ (ICSI) in women $>39$ years is acceptable, bearing in mind that the data for women receiving donor eggs were captured according to the donor age. Conversely, this may have led to an inflation of numbers in the younger age group.

Female age was inversely related to number of embryos/blastocysts transferred. Most women aged $\geq 40$ years received 3 embryos/ blastocysts, while in the 2 younger age categories, 2 was the most common number transferred. In all age groups, single and multiple embryo/blastocyst transfers took place. The transfer of more embryos/blastocysts, especially in older women, aims to optimise the chance of conception with acceptance of the risk of multiple pregnancy. Documenting the extent of this risk is an important future task for SARA.

Our first report has limitations. Firstly, not all South African ART units participated; although our findings represent approximately $70 \%$ of ART activity, they cannot be extrapolated to all ART treatment in the country. Secondly, the data submitted were accepted in good faith and were only tested for mathematical correctness. Although it is unlikely, discrepancies may exist between the reported and actual clinical data. Data inflation poses no conceivable benefit to the units; confidentiality and anonymity of the individual data were protected, and only pooled data were analysed and reported. Futhermore, based on our in-depth knowledge of ART in South Africa, we consider the results of the pooled data to be plausible.

This report documents CPR - rather than live birth rate (LBR) - as a marker of efficacy. LBR (increasingly singleton LBR) is the preferred indicator of ART success; ${ }^{12}$ however, many South African ART units do not follow pregnant patients beyond the first ultrasound (performed at 6 - 8 weeks of gestation when fetal heart activity and number of viable embryos can be detected). This is because the patients usually enter private or public obstetric services elsewhere. Similarly, the report does not document the rate of multiple pregnancy and birth, which is of central interest to ART data-monitoring. A relatively high CPR may be achieved through the practice of transferring several embryos/blastocysts; however, this risks twin and higher multipleorder pregnancies. The associated morbidity and mortality for fetuses and the mother has been established. Furthermore, multiple pregnancies are costly to health services and are a major factor in 
discussions concerning government and private insurance funding of ART. Given the importance of live and multiple births to all stakeholders, we intend to report these outcomes from 2012.

Additional information missing from this report includes the indication for treatment, number of egg donations, number and outcome of frozen embryo transfers, and ART complications. We intend to include this information in a more extended data capture commencing in 2012, using software developed with the South American Register for ART. Although SARA was designed for South Africa, its format is aligned to the South American register, thereby facilitating future comparison of data. Countries of South America have social, political and economic similarities to South Africa; therefore, this region is important as a benchmark against which to evaluate South African data. SARA's adherence to internationally defined and standardised recordings of ART will, however, allow comparison with any country or region, especially once SARA reports to international ART registers.

\section{Conclusion}

Despite its limitations, the first report on ART monitoring in South Africa contains valuable information for many stakeholders: (i) couples requiring ART can gauge their individual chance of success, as advised by the treating doctor, against a national average and age-specific CPR; (ii) ART units can compare their performance with a national benchmark; (iii) healthcare funders and planners have insight into the number of ART procedures and outcomes, and the extent to which ART demand is met in our country; and (iv) the general public is informed that ART is well established, with monitored and reported outcomes.

Contributions. The following units participated: Cape Fertility Clinic (Drs P le Roux, S Heylen, K Wiswedel, S Nosarka, R Dhansay, Ms K Raja); Care Clinic (Dr A Ramdeo, Mr K Naidoo); Drs Aevitas Incorporated (Profs/Drs T Kruger, I Siebert, $\mathrm{K}$ van der Merwe, Dr M Windt de Beer); Durban Fertility Clinic (Drs S Naidu, M Bhana, Mr N Moodley); Genesis Reproductive Centre (Drs A de
Bruin, J Pentz, G Hogewind, Mr J Lourens); Medfem Clinic (Drs J van Rensburg, J van Schouwenburg, T Rodrigues, A Esterhuizen); Parklane Fertility Centre (Dr H Netshidvhani, Ms S Karadasli); Port Elizabeth Infertility Unit (Drs P Dalmeyer, D Botha, Ms M Rijsdijk); Pretoria Fertility Clinic (Dr M Trouw, Mrs E Prinsloo); Reproductive Medicine Unit, Groote Schuur Hospital (Profs/Drs S Dyer, Z van der Spuy, T Matebese, Mrs M Vienings); Sandton Fertility Centre (Drs G Mohamed, M Faesen, R Patel, Ms K Raja); and Vitalab Fertility Unit (Drs S Volschenk, M Jacobson, L Gobetz, Ms J Meintjies).

Acknowledgements. We gratefully acknowledge the valuable assistance from the SASREG committee, SASREG secretariat (representing Turner's Conferences) and members of the Latin American Register for ART. Ferring and Merck-Serono provided financial support for national meetings to discuss data collection. We thank all patients who placed their trust in the clinical teams and ART.

\section{References}

1. Collins JA. Cost-effectiveness of in vitro fertilization. Semin Reprod Med 2002;19:279-289.

2. Germond M, Wirthner D, Senn A. Core data for assisted reproductive technology registers: results of a consensus meeting. Reprod Biomed Online 2008;17(6):834-840.

3. Nygren KG, Sullivan E, Zegers-Hochschild F, et al. ICMART World Report: Assisted Reproductive Technology 2003. Fertil Steril 2011;95(7):2209-2222.

Zegers-Hochschild F, Adamson GD, de Mouzon J, et al. ICMART/WHO revised glossary on ART terminology, 2009. Hum Reprod 2009;24(11):2683-2687.

terme 5. De Mouzon J, Goossens V, Bhattacharya S, et al. Assisted reproductive technology in Europe
results generated from European registers by ESHRE. Hum Reprod 2010;25(8):1851-1862.

results generated from European registers by ESHRE. Hum Reprod 2010;25(8):1851-1862.
6. Luceno F, Castilla JA, Gomez-Palomares JL, et al. Comparison of IVF cycles reported in a voluntary 6. Luceno F, Castilla JA, Gomez-Palomares JL, et al. Comparison of IVF cycles reported
ART registry with a mandatory registry in Spain. Hum Reprod 2010;25(12):3066-3071.

7. Gunby J, Bissonnette F, Librach C, Crowan L. Assisted reproductive technologies in Canada: 2007 results from the Canadian ART register. Fertil Steril 2011;95(2):547.el-547.e10

8. ESHRE Capri Workshop Group. Social determinants of human reproduction. Hum Reprod 2001;16(7):1518-1526.

9. Nachtigall RD. International disparities in access to infertility services. Fertil Steril 2006;85(4):871-875. 10. Statistics South Africa. Census 2001 Key Results. Pretoria: Statistics South Africa. http://www.statssa. gov (accessed 30 August 2011).

1. Mouzon J, Lancaster P, Nygren KG, et al. World collaborative report on assisted reproductive technology 2002. Hum Reprod 2009;24(9):2310-2320.

12. Schieve LA, Reynolds MA. What is the most relevant standard of success in assisted reproduction? Hum Reprod 2004;19(4):778-782.

Accepted 31 October 2011 